\title{
An Optimized Control Method for Four-Leg Inverter
}

\author{
$\mathrm{Xu} \operatorname{Jin}^{1,2, *}$, Li Lin ${ }^{1}$ and Zhao Sanqin ${ }^{1,2}$ \\ ${ }^{I}$ College of Engineering, Nanjing Agricultural University, China \\ ${ }^{2}$ The Key Laboratory in the Aspect of Intelligent Agricultural Equipment, Jiangsu Province, China
}

\begin{abstract}
This paper studies three-phase four-leg inverter and conducts the system analysis under the situations of balanced and unbalanced load. Based on existing decoupling control method, this paper analyses the principle of decoupling control method and presents a new optimized digital control method. This optimized method uses the last calculation result to compensate for the error and simplifies the control. It only needs to sample the neutral line current whereas the voltage sampling of load is not necessary. This paper conducts simulation and experiment to verify the control method, and the performance is perfect.
\end{abstract}

Keywords: Decoupling control, four-leg Inverter, unbalanced load.

\section{INTRODUCTION}

In the design scheme of the three-phase four-wire system out put inverter, the structure of four-leg has been widely used for the advantages of the low utilization of direct voltage, the strong ability with unbalanced load, the flexible methods of control and the number of power devices used etc. The structure has been widely used in asymmetrical three-phase load or the nonlinear load, to balance the threephase current and it will generate the unbalanced three-phase voltage [1]. In this system structure, the function of the fourth bridge is to make the midpoint voltage of three-phase load, zero by controlling the neutral line current, so as to maintain the voltage of three-phase load equilibrium [2]. Line inductance series has to be maintained in the midline for controlling the neutral current, but the control of the three-phase four-wire system became very complicated, as the neutral current is the sum of three-phase current and the midline inductor will be coupled with three-phase filter inductor [3]. At present, there are mainly four kinds of control methods: decoupling control, the SVM control, carrier PWM control, error judgment control method. Aiming at decoupling control method, this paper puts forward an optimized control strategy which will improve the control effect of four-leg [4-9].

\section{THREE-PHASE EQUILIBRIUM SYSTEM ANALY- SIS}

Fig. (1) is the schematic diagram of three-phase four-leg inverter. The circuit is composed of three-phase bridge arms A, B, C and neutral line bridge arm. The capacity values of

*Address correspondence to this author at the College of Engineering, Nanjing Agricultural University, China; Tel: 025-58606585;

Fax:025-58606585; E-mail: xujin@njau.edu.cn the two capacitances on the DC bus are equal and their halfway point is selected as reference ground. Three-phase outputs use the LC smoothing, and the filter inductors are, respectively, the midline inductor is $\mathrm{L}_{\mathrm{N}}$, the voltage of the midpoint load is $\mathrm{M}$, the voltages of the three-phase loads are shown as follows:

The switch state function is defined as Eq. (1).

$$
S_{i}=\left\{\begin{array}{ll}
1 & \mathrm{~S}_{i 1} \mathrm{ON}, \mathrm{S}_{i 2} \mathrm{OFF} \\
0 & \mathrm{~S}_{i 1} \mathrm{OFF} \mathrm{S}_{i 2} \mathrm{ON}
\end{array} \quad i=\mathrm{A}, \mathrm{B}, \mathrm{C}, \mathrm{N}\right.
$$

When $\mathrm{S}$ is zero, the output of $\mathrm{i}$ bridge arm is 0.5 , when $\mathrm{S}$ is one, the output of $i$ bridge arm is -0.5 . Therefore, the difference values of the each bridge arm output voltage and neutral line bridge arm is Eq. (2).

$u_{i \mathrm{~N}}=\left(S_{i}-S_{\mathrm{N}}\right) V_{\mathrm{dc}} \quad i=\mathrm{A}, \mathrm{B}, \mathrm{C}$

According to KVL law, we derived Eq. (3).

$$
\left\{\begin{array}{l}
u_{\mathrm{AN}}=u_{\mathrm{A}}-u_{\mathrm{N}}=L_{\mathrm{A}} \frac{d i_{\mathrm{A}}}{d t}+u_{\mathrm{AM}}+L_{\mathrm{N}} \frac{d i_{\mathrm{N}}}{d t} \\
u_{\mathrm{BN}}=u_{\mathrm{B}}-u_{\mathrm{N}}=L_{\mathrm{B}} \frac{d i_{\mathrm{B}}}{d t}+u_{\mathrm{BM}}+L_{\mathrm{N}} \frac{d i_{\mathrm{N}}}{d t} \\
u_{\mathrm{CN}}=u_{\mathrm{C}}-u_{\mathrm{N}}=L_{\mathrm{C}} \frac{d i_{\mathrm{C}}}{d t}+u_{\mathrm{CM}}+L_{\mathrm{N}} \frac{d i_{\mathrm{N}}}{d t}
\end{array}\right.
$$

Under the stable state, the voltage of the three-phase load is balanced. The relation between output of the neutral line bridge arm, and the midpoint of the load voltage is shown in the Eq. (4):

$$
\left\{\begin{array}{l}
u_{\mathrm{AM}}=U_{\max } \sin (\omega \mathrm{t}) \\
u_{\mathrm{BM}}=U_{\max } \sin \left(\omega \mathrm{t}-\frac{2 \pi}{3}\right) \\
u_{\mathrm{CM}}=U_{\max } \sin \left(\omega \mathrm{t}+\frac{2 \pi}{3}\right)
\end{array}\right.
$$




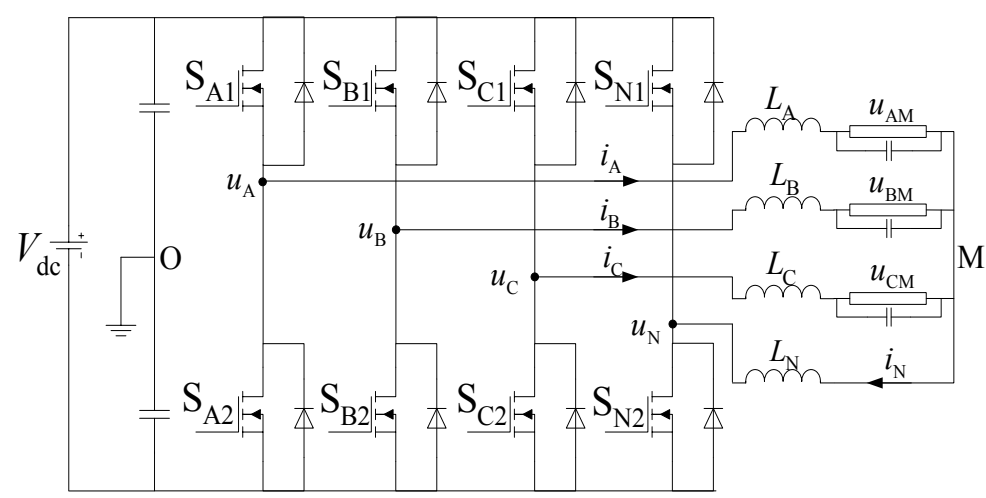

Fig. (1). Diagram of four-leg inverter.

Now the output voltages of the three-phase bridge arms are given in Eq. (5).

$\left\{\begin{array}{l}u_{\mathrm{A}}=L_{\mathrm{A}} \frac{d i_{\mathrm{A}}}{d t}+u_{\mathrm{AM}} \\ u_{\mathrm{B}}=L_{\mathrm{B}} \frac{d i_{\mathrm{B}}}{d t}+u_{\mathrm{BM}} \\ u_{\mathrm{C}}=L_{\mathrm{C}} \frac{d i_{\mathrm{C}}}{d t}+u_{\mathrm{CM}}\end{array}\right.$

If the unbalance load is symmetrical, three-phase currents are also symmetrical. Therefore, three-phase modulation waves are also symmetrical when the PWM waveform is produced to control the three-phase bridge arm, and the vector synthesis of three-phase modulation wave vector is a circle. But when the three-phase is asymmetric, the three-phase load voltage and current are no longer symmetrical; the synthesis of three-phase modulation wave vector becomes the oval. Thus, it is unable to realize the three-phase independent control by controlling the neutral line bridge arm.

\section{SYSTEM ANALYSIS OF THREE-PHASE IMBAL- ANCE STATE}

When the three-phase is imbalance, and the midpoint of three-phase load M's potential is not zero, then the Eq. (5) turns into Eq. (6).

$\left\{\begin{array}{l}u_{\mathrm{A}}=L_{\mathrm{A}} \frac{d i_{\mathrm{A}}}{d t}+u_{\mathrm{AM}}+u_{\mathrm{M}} \\ u_{\mathrm{B}}=L_{\mathrm{B}} \frac{d i_{\mathrm{B}}}{d t}+u_{\mathrm{BM}}+u_{\mathrm{M}} \\ u_{\mathrm{C}}=L_{\mathrm{C}} \frac{d i_{\mathrm{C}}}{d t}+u_{\mathrm{CM}}+u_{\mathrm{M}}\end{array}\right.$

In order to meet $=0$, we get Eq. (7).

$u_{\mathrm{N}}=u_{\mathrm{M}}-L_{\mathrm{N}} \frac{d i_{\mathrm{N}}}{d t}$

Assuming that three-phase filtering inductance is equal, we get Eq. (8).
$L_{\mathrm{A}}=L_{\mathrm{B}}=L_{\mathrm{C}}=L$

From Eq. (6), we acquire Eq. (9).

$u_{\mathrm{A}}+u_{\mathrm{B}}+u_{\mathrm{C}}=u_{\mathrm{AM}}+u_{\mathrm{BM}}+u_{\mathrm{CM}}+3\left(L \frac{d\left(i_{\mathrm{A}}+i_{\mathrm{B}}+i_{\mathrm{C}}\right)}{d t}+u_{\mathrm{M}}\right)$

If the three-phase control is independent, the three-phase modulation waves will reach three-phase equilibrium. So, at the same time it satisfies the condition. Thus, Eq. (7) and Eq. (9) will give Eq. (10)

$u_{\mathrm{N}}=-\frac{1}{3}\left(u_{\mathrm{AM}}+u_{\mathrm{BM}}+u_{\mathrm{CM}}\right)-\left(\frac{L}{3}+L_{\mathrm{N}}\right) \frac{d i_{\mathrm{N}}}{d t}$

The Eq. (10) is the output voltage of the neutral line bridge arm when the three-phase load is imbalance.

\section{THE OPTIMIZED DIGITAL CONTROL METHOD}

In the last section, we have discussed the output voltage of the neutral line bridge arm under the imbalance load. From Eq. (10) it can be seen that the control of neutral line voltage is complex as it involves differential operation, so we choose a digital control method to control. At the same time, consider that the Eq. (10) contains differential operation, when faced with a phase change in load, $\frac{d i_{\mathrm{N}}}{d t}$ will become bigger; It will affect the stability of the system, so we need to transform differential operation into integral operation. Because the result of the integral operation lags $180^{\circ}$ behind differential operation, and the amplitude is of differential operation, so we use it in place of differential operation. Thus, Eq. (11) is obtained.

$u_{\mathrm{N}}=-\frac{1}{3}\left(u_{\mathrm{AM}}+u_{\mathrm{BM}}+u_{\mathrm{CM}}\right)+\left(\frac{L}{3}+L_{\mathrm{N}}\right) \omega^{2} \int i_{\mathrm{N}} d t$

Let $\frac{L}{3}+L_{\mathrm{N}}=\alpha$, and put Eq.(11) into discretization to find Eq. (12).

$u_{\mathrm{N}}(k)=-\frac{1}{3}\left[u_{\mathrm{AM}}(k)+u_{\mathrm{BM}}(k)+u_{\mathrm{CM}}(k)\right]+\alpha \omega^{2} \sum T_{s} i_{\mathrm{N}}(k)$ 


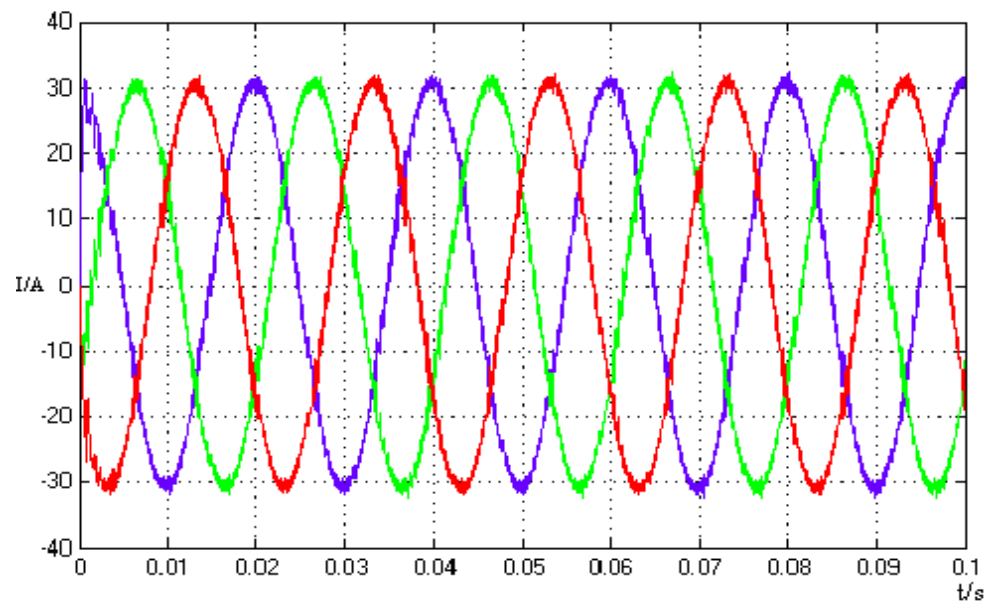

Fig. (2). The output current waveforms of inductance when three-phase loads are balanced.

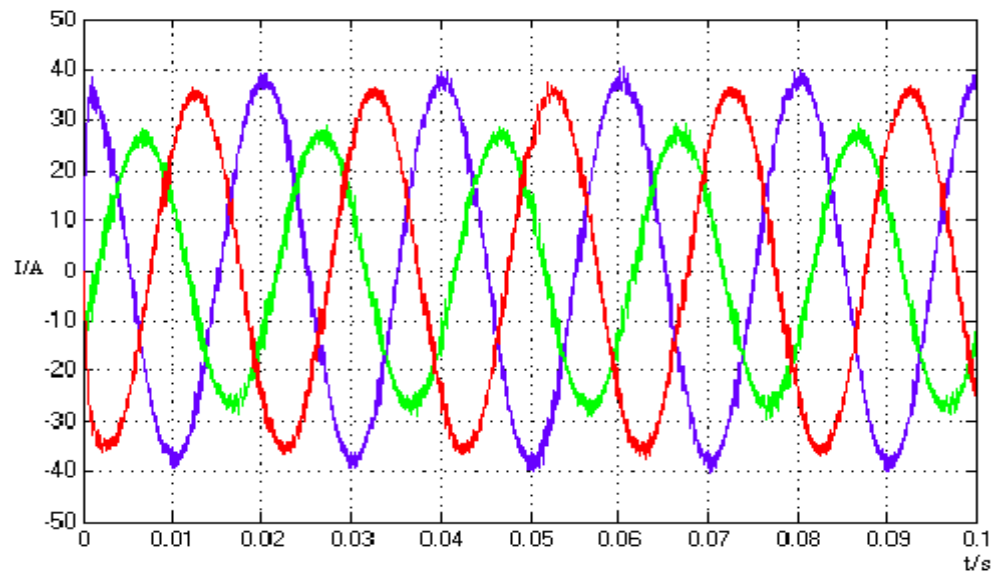

Fig. (3). The output current waveforms of inductance when three-phase loads are imbalanced.

Under the actual circumstances, there will always be errors between differential and integral calculations, in order to compensate the error, we optimize Eq. (12) to require Eq. (13)

$u_{\mathrm{N}}(k)=\beta(k)\left[u_{\mathrm{AM}}(k)+u_{\mathrm{BM}}(k)+u_{\mathrm{CM}}(k)\right]+\alpha \omega^{2} \sum T_{s} i_{\mathrm{N}}(k)$

The Eq. (13) compensates the errors by multiplying a coefficient of the sum of three-phase voltages. We need the real optimization to improve the accuracy of the compensation. Assuming if the coefficient is continuous that the last calculation of and $u_{\mathrm{N}}(k)$ is not mutated, then we can use the last calculation results to optimize, just like the Eq. (14) shows.

$\beta(k)=\frac{u_{\mathrm{N}}(k-1)-\alpha \omega^{2} \sum T_{s} i_{\mathrm{N}}(k)+\alpha\left[\frac{i_{\mathrm{N}}(k)-i_{\mathrm{N}}(k-1)}{T s}-\omega^{2} \sum T_{s} i_{\mathrm{N}}(k)\right]}{\left[u_{\mathrm{AM}}(k)+u_{\mathrm{BM}}(k)+u_{\mathrm{CM}}(k)\right]}$

The Eq. (14) uses the previous calculations to optimize the coefficient, and compensate the error of differential and integral calculations. We can get Eq. (15) by rearranging Eq. (14).
$\beta(k)=\frac{u_{\mathrm{N}}(k-1)-\alpha\left[2 \omega^{2} \sum T_{s} i_{\mathrm{N}}(k)-\frac{i_{\mathrm{N}}(k)-i_{\mathrm{N}}(k-1)}{T_{s}}\right]}{u_{\mathrm{AM}}(k)+u_{\mathrm{BM}}(k)+u_{\mathrm{CM}}(k)}$

Then, we take Eq. (15) to Eq. (13) for substitution.

$u_{\mathrm{N}}(k)=u_{\mathrm{N}}(k-1)+\alpha\left[\frac{i_{\mathrm{N}}(k)-i_{\mathrm{N}}(k-1)}{T_{s}}-\omega^{2} \sum T_{s} i_{\mathrm{N}}(k)\right]$

The Eq. (16) is the final output voltage of neutral line bridge arm; this method can control the output voltage of the neutral line bridge arm through sampling the current on the central line. It greatly simplifies the control algorithm without having anything to do with the voltage of load, and realizes the decoupling control.

\section{SIMULATION AND EXPERIMENT}

\subsection{The Result of Simulation}

The simulation model is set up as follows; simulation parameter is $\mathrm{DC} 600 \mathrm{~V}$, the output filter inductance is $3 \mathrm{Mh}$, the filter capacitor is $10 \mathrm{uF}$, the switching frequency is $10 \mathrm{KHz}$. 


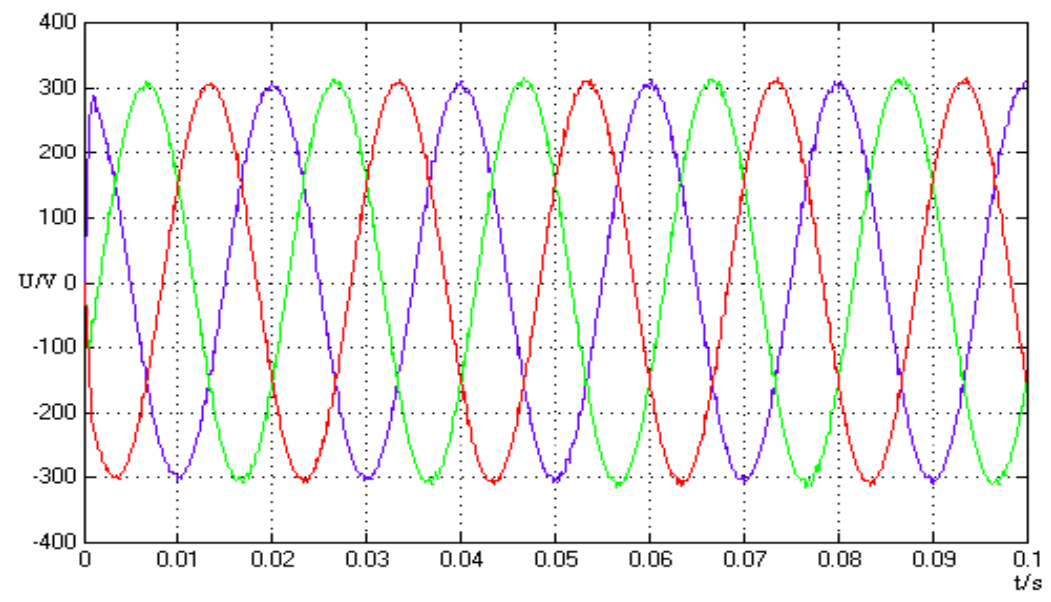

Fig. (4). The output voltage waveforms of inductance when three-phase loads are imbalanced.

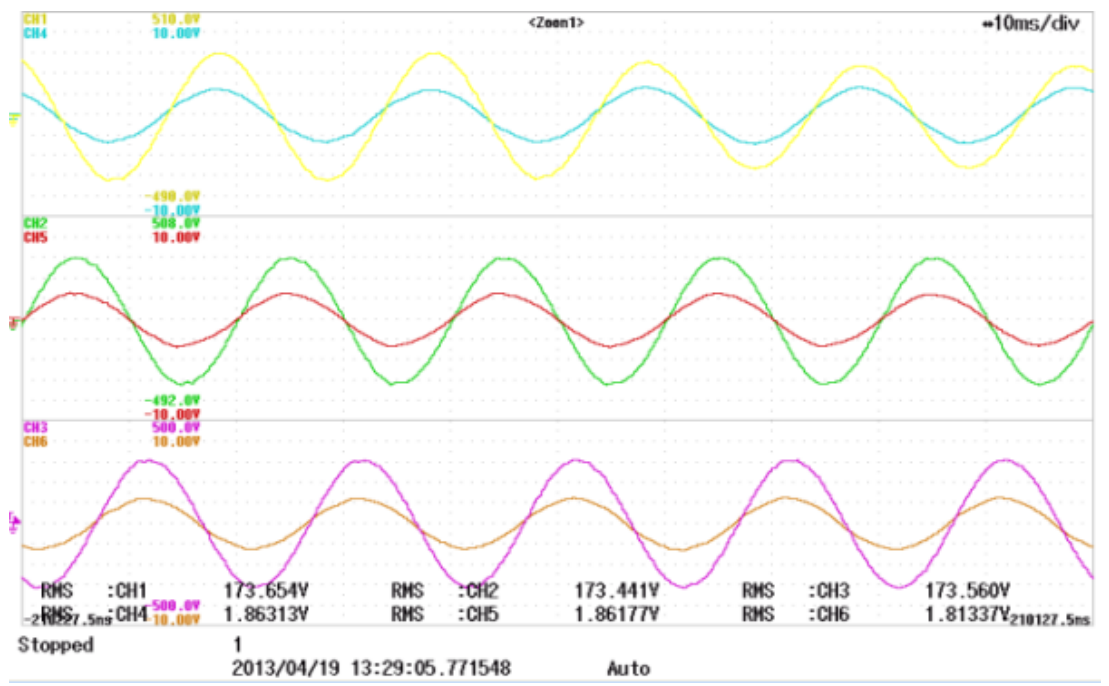

Fig. (5). The output voltage and current waveforms of the loads when the three-phases are balanced.

When the load is unbalanced the imbalance rate is: phase $\mathrm{A}$ is $20 \%$, phase B is $30 \%$. From the waveform analysis of Fig. (2), Fig. (3) and Fig. (4) we can find that the output voltages are always maintain symmetry when the loads

are imbalance, and it shows that the simulation algorithm is feasible.

\subsection{Experiment Result}

In order to further verify this control method proposed, we set up an off-grid three-phase four-leg inverter experiment platform on the basis ofTMS320F28335. The parameter of this $50 \mathrm{kw}$ three-phase four-leg inverter is: WPAP 330150 AC network AVDD and $150 \mathrm{kwDC}$ AVDD. Its maximum DC and AC power are both $150 \mathrm{kw}, \mathrm{DC}$ open-circuit voltage is $1000 \mathrm{v}$. YOKOGAWADL850 oscillo graphic recorder has $100 \mathrm{MS} / \mathrm{s}$ speed, and high resolution of 16 bits, and isolation of $1 \mathrm{kV}$.

Fig. (5) shows the output waveforms of the three-phase voltages and currents when each phase of three-phase loads is $15 \mathrm{KW}$. It obvious that three-phase voltage and current maintain symmetry and each phase has $120^{\circ}$ difference with the other. The Fig. (6) shows the waveforms of voltages and currents for unbalanced loads; at the beginning, A, B and C of three-phase loads are all $15 \mathrm{KW}$, and then $\mathrm{A}$ and $\mathrm{C}$ remain unchanged; the load of phase $\mathrm{B}$ is changed from the original $15 \mathrm{kw}$ to $6 \mathrm{kw}$, the current of phase $\mathrm{B}$ is changed from the original $68 \mathrm{~A}$ to $27.3 \mathrm{~A}$. The diagram further shows that threephase voltage amplitudes and phases remain unchanged when the loads switch over. The analysis that when the three-phase is imbalance, the three-phase output inductor currents will be asymmetrical, and there will be a current flowing through the fourth bridge arm. Thus, we will achieve the expected control effect. Through the analysis of experiment results, it is verified that the three-phase four-leg inverter has the ability to carry unbalanced loads. To put it another way, it can be established that the three-phase fourleg inverter is stronger in stability that it can still give output symmetrical three-phase voltages under the condition of unbalanced loads. 


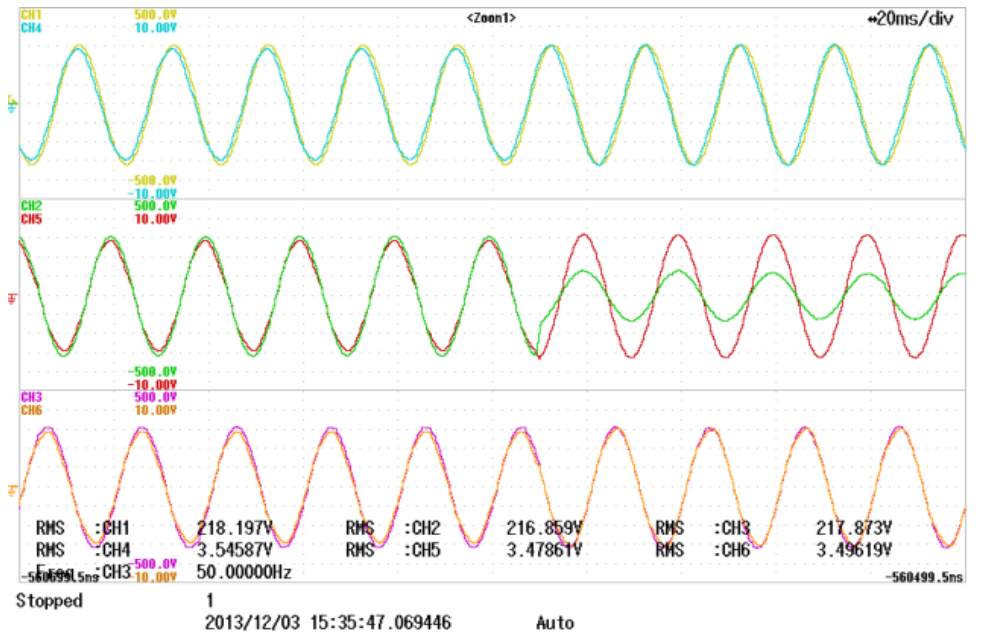

Fig. (6). The output voltage and current waveforms of the loads when the three-phases are imbalanced.

\section{CONCLUSION}

This paper analyzes the decoupling control algorithm of the three-phase four-leg inverter, and optimizes the decoupling algorithm. The optimized method uses the last result to compensate the error of current calculation. This method only needs to sample the neutral line current and the voltage sampling of load is not necessary; and it simplifies the control. This paper also conducts simulation and experiment to verify the control method, and the performance is perfect.

\section{CONFLICT OF INTEREST}

The authors confirm that this article content has no conflict of interest.

\section{ACKNOWLEDGEMENTS}

The authors would like to thank Dr. Lin Li for preliminary investigations on the topic of grid Inverter.

\section{REFERENCES}

[1] R. Zhang, V. H. Prasad, D. Boroyevich, and F.C. Lee, "Threedimensional space vector modulation for four leg voltage-source converters," IEEE Transactions on Power Electronics, vol. 17, no. 3, pp. 314-326, 2002.

[2] O. Ojo, and P. M. Kshirsagar, "Concise modulation strategies for four-leg voltage source inverters," IEEE Transactions on Power Electronics, vol. 19, no. 1, pp. 35-40, 2004.

[3] H. Wang, Y. Ding, F. Zhang, X. Chen, and Y. Yan, "Midline inductor of four-leg three-phase inverter based on switching-node preset," Proceedings of the CSEE, vol. 28, no. 9, pp. 23-28, 2008.

[4] M. J. Ryan, D. R. W. De, and R. D. Lorenz, "Decoupled control of a four-leg inverter via a new 44 transformation matrix," IEEE Transactions on Power Electronics, vol. 16, no. 5, pp. 694-701, 2001.

[5] X. C. Liu, H. G. Zhang, and H.Z. Cheng, "The control strategy of the fourth bridge arm in four-leg inverter," Proceedings of the CSEE, vol. 27, no. 33, pp. 87-92, 2007.

[6] H. Z. Chen, and X. C. Liu, "The decoupling control of the four-leg three-phase inverter," Proceedings of the CSEE, vol. 27, no. 19, pp. 74-79, 2007.

[7] X.Y. Zhang, Control and Design of the Three-Phase Four-Leg Inverter, Xihua University: Chengdu, 2011.

[8] C. Han, "The Research on the Three-Phase Four-Leg Inverter Power Supply Control," Qinhuang Dao: Yanshan University, 2007.

[9] C.Y. Gong, X. Yu, M. Li, X. Cheng, and Y.G. Yan, "The threedimensional space vector control technology research of the threephase four-leg inverter," Transactions of China Electrotechnical Society, vol. 19, no. 12, pp. 30-36, 2004.

(C) Jin et al.; Licensee Bentham Open.

This is an open access article licensed under the terms of the Creative Commons Attribution Non-Commercial License (http://creativecommons.org/licenses/by$\mathrm{nc} / 4.0 /$ ) which permits unrestricted, non-commercial use, distribution and reproduction in any medium, provided the work is properly cited. 